\title{
Erratum: Gravitational waves from the quasicircular inspiral of compact binaries in Einstein-aether theory [Phys. Rev. D 101, 044002 (2020)]
}

Chao Zhang, Xiang Zhao, Anzhong Wang®, Bin Wang, Kent Yagi, Nicolas Yunes, Wen Zhao, and Tao Zhu

(C) (Received 27 August 2021; published 16 September 2021)

DOI: $10.1103 /$ PhysRevD.104.069905

Some errors with Eqs. (6.11) and (6.14) occur. In particular, Eq. (6.11) should read

$$
A_{p p E}^{(l, N)}(f)=A_{G R}^{(2)}\left[\rho_{p p E}^{(N)}+\left(\mathcal{U}_{l}\right)^{\bar{a}_{p p E}^{(l)}} \sum_{k=0}^{\infty} \bar{\alpha}_{p p E, k}^{(l, N)}\left(\mathcal{U}_{l}\right)^{k}\right]
$$

Accordingly, Eq. (6.14) should be

$$
A_{p p E}^{(2, N)}(f)=A_{G R}^{(2)}\left[\rho_{p p E}^{(N)}+\left(\mathcal{U}_{2}\right)^{\bar{a}_{p p E}^{(2)}} \bar{\alpha}_{p p E, 0}^{(2, N)}\right]
$$

here, the $\rho_{p p E}^{(N)}$ 's read

$$
\begin{aligned}
\rho_{p p E}^{(T)} & =\kappa_{3}^{-1 / 2}, \\
\rho_{p p E}^{(S)} & =\kappa_{3}^{-1 / 2} \frac{g_{b 1} F_{b} \sin ^{2} \vartheta+g_{L 1} F_{L} \sin ^{2} \vartheta}{g_{+} F_{+}\left(1+\cos ^{2} \vartheta\right)-i g_{\times} F_{\times} \cos \vartheta}, \\
\rho_{p p E}^{(V)} & =\kappa_{3}^{-1 / 2} \frac{g_{X 1} F_{X} \sin (2 \vartheta)-i g_{Y 1} F_{Y} \sin \vartheta}{g_{+} F_{+}\left(1+\cos ^{2} \vartheta\right)-i g_{\times} F_{\times} \cos \vartheta} .
\end{aligned}
$$

With these corrections, all the 0th-PN terms are kept, and our major results, i.e., Eqs. (6.17) and (6.18), remain the same.

In addition, a typo in Eq. (3.29) happens, in which $\beta_{2}$ should be written as

$$
\beta_{2} \equiv \frac{c_{14}-2 c_{+}}{2 c_{14}\left(1-c_{+}\right) c_{S}^{2}} \text {. }
$$

Note that this is simply a typo that does not propagate, and as a result, nothing else is affected. 